Volume 8, No.3, April - May 2019

International Journal of Wireless Communications and Networking Technologies

Available Online at http://warse.org/IJWCNT/static/pdf/file/ijwent05832019.pdf

https://doi.org/10.30534/ijwcnt/2019/05832019

\title{
Crowdsourced Pothole Mapping and Route Navigation
}

\author{
Johns Soney ${ }^{1}$ Jithin K George ${ }^{2}$ Bobby Isac ${ }^{3}$ Babilu Chummar Joshy ${ }^{4}$ Manoj T Joy ${ }^{5}$ \\ ${ }_{1}^{1}$ APJ Abdul Kalam Technological University, India, johnssoney@cs.ajce.in \\ ${ }^{2}$ APJ Abdul Kalam Technological University, India, jithinkgeorge@cs.ajce.in \\ ${ }^{3}$ APJ Abdul Kalam Technological University, India, bobbyisac@cs.ajce.in \\ ${ }^{4}$ APJ Abdul Kalam Technological University, India, babiluchummarjoshy@cs.ajce.in \\ ${ }^{5}$ APJ Abdul Kalam Technological University, India, manojtjoy@amaljyothi.ac.in
}

\begin{abstract}
The proposed system aims to create a crowdsourced map of potholes on public roads. Users can report a pothole along with pictures and location. These reports can be viewed by other users as well as concerned authorities, to take necessary actions. Here, we also use machine learning techniques to identify false reports. Computer vision techniques can be implemented to verify potholes in the uploaded images, using edge detection. Once the roads are repaired, the corresponding potholes can be removed by the authorities. Users can also use the app to navigate to a certain location by avoiding bad roads.
\end{abstract}

Key words: Android, Crowd, Machine Learning

\section{INTRODUCTION}

\section{A. Background}

With the increase in population, there has been an increase in the number of vehicles on the road. Roads are getting crowded day by day. As a result, potholes start to form in roads makes the road difficult to drive upon. Potholes took a deadly toll in 2017, claiming almost 10 lives daily with annual fatalities in the country adding up to 3,597 - a more than 50

\section{B. Motivation}

"Father fills up 556 potholes after his son's death."

Almost three years after he lost his 16 year old son to a pothole on a road at Jogeshwari-Vikhroli Link Road Mumbai, Dadarao Bilhore fills up potholes at the same spot as a tribute to his son, Prakash Bilhore. His sister-in-law was also injured due to a pothole. Bilhore filled up 556 potholes and he is looking forward to continue doing so, as he did not want another life to be lost to a similar incident. "I have realized that the authorities work only when an accident like this happens, but question is why wait for something to happen?", says Bilhore. Ever since the fatal accident, Bilhore has taken it upon himself to fill up even small potholes when he spots them. [1]

\section{ANDROID APP DEVELOPMENT}

\section{A. Home Screen-Map View}

The app begins by showing the google map, thus locating the various potholes around the users current location. The user can navigate to certain location without signing in and the user can report pothole by signing in to the app (Figure. 1.)
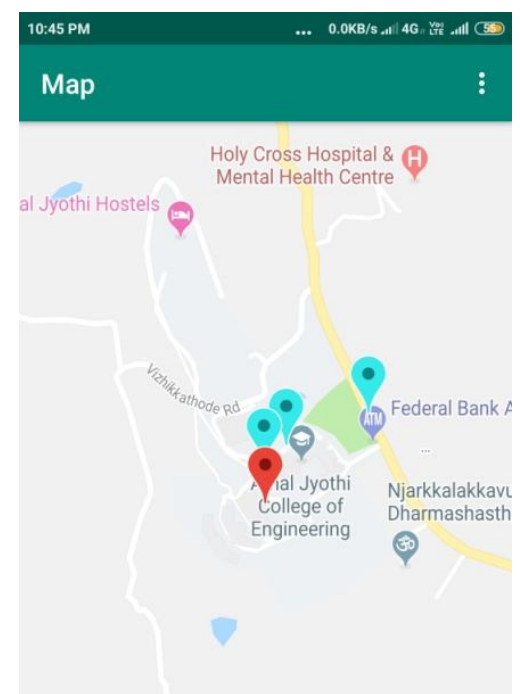

Figure 1: Map View

\section{B. Reporting a new pothole}

Users can report pothole by signing in to the app and choosing the option 'Report pothole' as shown in Figure 2.

\section{Searching Location}

Users can search the location for which they want to reach the destination through roads with least potholes.(Figure 3.) 


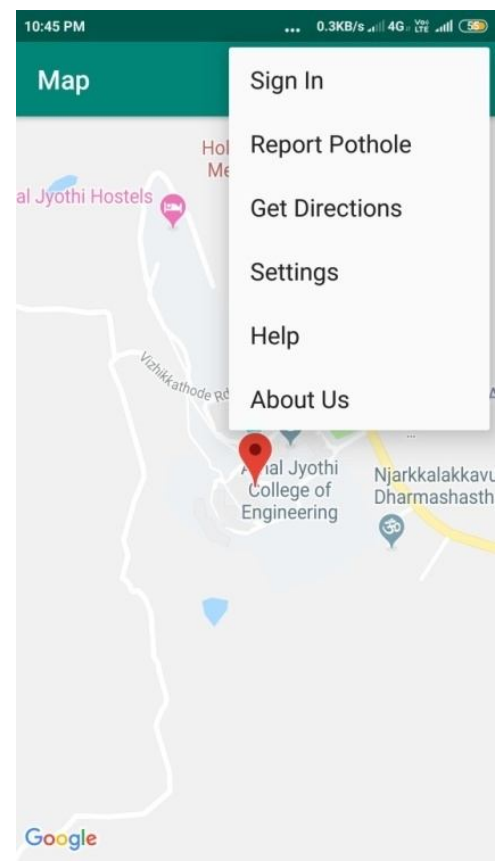

Figure 2: Report pothole

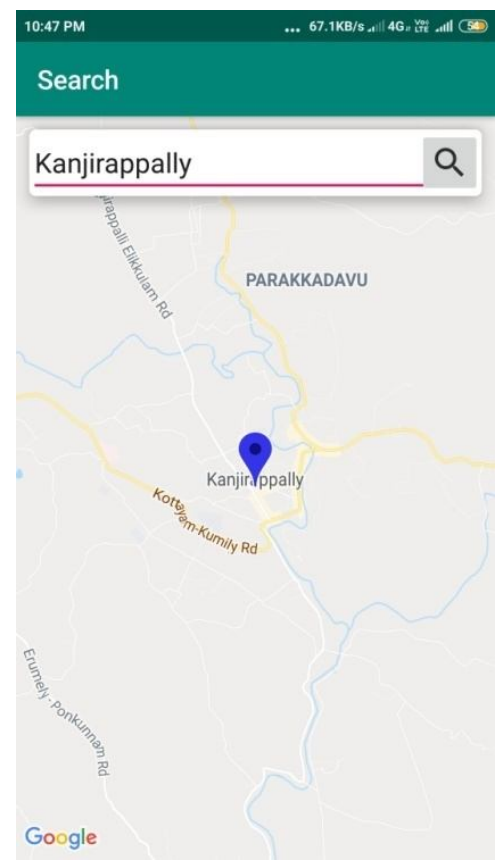

Figure 3: Taking image and fetching location to add it to database.

\section{BACKEND Web SERVER}

Data processing is almost completely handled by the Web Server. We are using Firebase as the primary platform and infrastructure provider for our backend. Authentication and app analytics are provided by Firebase APIs. This is enables us to have user friendly approaches like single sign-on and 'sign-in with Google' options. One major area of concern for a crowdsourced platform is a robust database. We are relying on Firebase Cloud Firestore, their latest and best Real Time Database to date. Firestore enables our application to reflect changes to the data in real time, as soon as the data is changed in the database. For examples, as soon as a user reports a pothole to the system, another user who had the app open already will see the new pothole appear on his screen. Besides the database, we use Firebase Cloud Functions to host our code. These codes are used to validate the data, call the machine learning component and to manipulate the data. For example, as soon as someone reports a pothole as repaired, a notification is sent to all users in that particular area and if most of them accept the change, it will be updated to the database. This validation is done using code hosted in the Cloud Functions. (Figure 4)

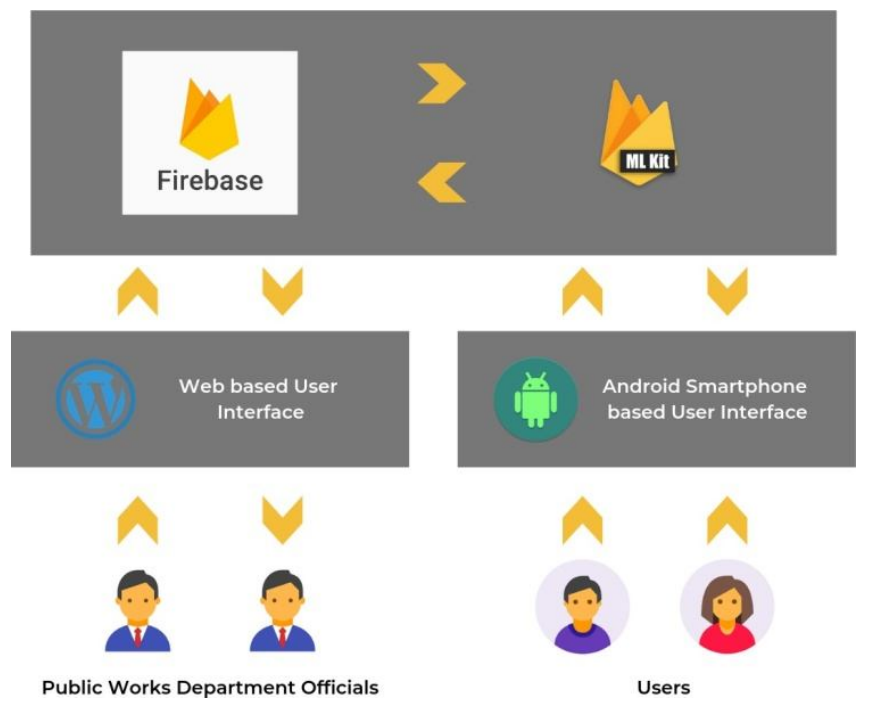

Figure 4.: Firebase

\section{A. User Authentication}

To report a pothole he/she has to sign in to the app where we upload the image of pothole and current geographical location. The back-end product 'Authentication' is used for users to sign up and sign in.

\section{B. Taking images of Pothole and fetching location}

After signing in, the user can click 'OPEN CAMERA' to take pictures and upload to firebase.(Figure 5.) 


\section{MACHINE LEARNING}

Machine Learning is used to detect false reports of potholes. The way our system works, the user has to take a picture of the pothole from the location itself. This image will be used for two validations: $i$. Whether the image is of a road or something else like a person, tree etc. ii. Whether the image of the road contains a pothole or not. For this, we are using Google's Vision API for image processing. We upload a dataset of more than 200 images which include images of true potholes, clean roads, roads littered with other elements, and finally some random images like trees, people and animals. The AutoML component of the Vision platform trains the model automatically, and for a labeling threshold value of 0.90 , we get the best values for precision $96.8 \%$ and accuracy $93.8 \%$.

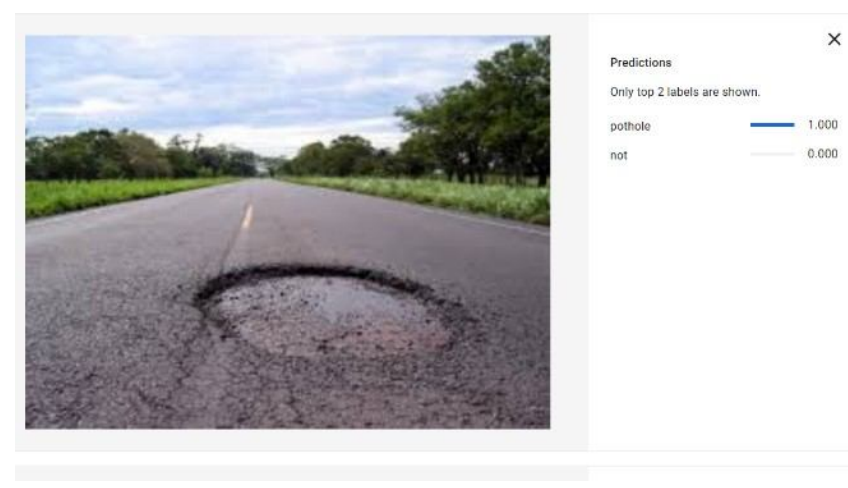

Figure 5: ML model identified pothole.

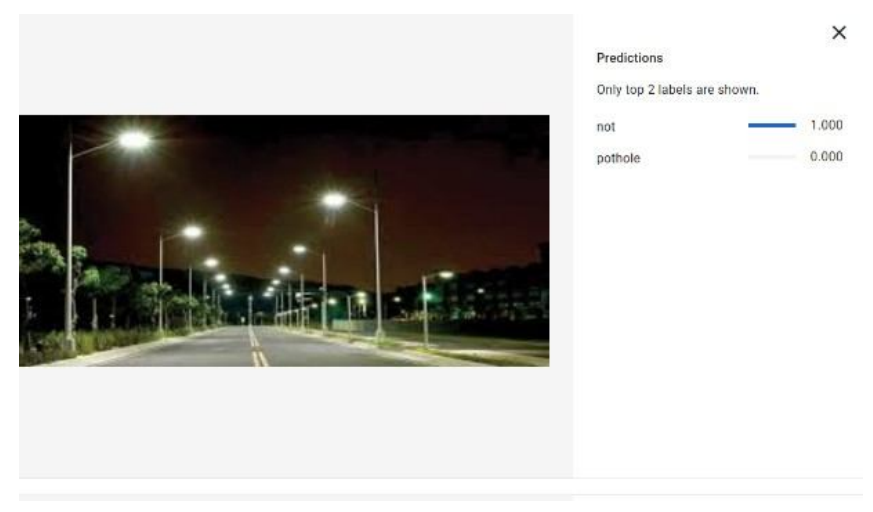

Figure 6: ML model didn't identify pothole.

\section{Web Dashboard}

\section{A. Admin and Government portal}

Here we have the platform for both admin and higher authorities to sign in to the web dashboard and allows them to take necessary actions. They can cross-check whether the users add correct details or not. The dashboard will contain all district wise list of potholes so that it will be easy for the admin to manage and authorities to control and take action.

\section{RELATED WORKS}

\section{A. Pothole detection system using black-box camera}

This proposed pothole detection system uses commercially available black box camera. The algorithms detect potholes over a large area in real time using computer vision techniques, including feature extraction, line detection, and threshold evaluation. The system uses cameras mounted on to the front of the car. [1]

\section{B. Monitoring Of Road irregularities using IOT}

This system uses an Arduino controlled sensor module that includes a GPS and Accelerometer. The accelerometer detects sudden jolts and bumps. The values from accelerometer can be used to detect severity of potholes. This is combined with GPS location coordinates to make a map of the potholes. This method relies on having specialized equipment attached to the car used for mapping the potholes. [2]

\section{The Pothole Patrol: Using a Mobile Sensor Network for Road Surface Monitoring}

Pothole Patrol system proposes a collection of sensors equipped vehicles, and associated algorithms for detecting potholes. The data is collected using vibration and GPS sensors, and the system was implemented on 7 taxis in the Boston area. [3]

\section{Pothole Detection System using Machine Learning on Android}

This system is similar to the ones above, as it uses mobile devices to record data. It uses accelerometers to detect anomalies and uses machine learning to remove false positives. The raw pothole data can also be saved as a .csv file so that it can be sent to any mail address. The neural network uses an Android machine learning framework called Encog. [4]

\section{E. Automated Management of pothole related disasters using image processing and geo-tagging}

Image processing is used for identification of target pothole regions in the $2 \mathrm{D}$ images using edge detection and morphological image processing operations. A method is developed to accurately estimate the dimensions of the potholes from their images, analyze their area and depth, estimate the quantity of filling material required and therefore enabling pothole attendance on a priority basis. This will further enable the government official to have a fully automated system for effectively managing pothole related disasters. [5]

\section{Future SCOPE}

Areas where this system can be improved is discussed here formerly and later in this section the areas where the improvised system can be made use is discussed. Right now the system manually sets the severity of the potholes, but by making use of Computer Vision techniques namely the edge and depth detecting algorithms we expect the system to automatically set the severity of the potholes. Eventually with large amount of data collected, the system predicts the 
Johns Soney et al., International Journal of Wireless Communications and Network Technologies, 8(3), April - May 2019, 21 - 24

roads which are most vulnerable to potholes. There are many areas where this system can be improvised and implemented. The system can be made useful to prevent or at least reduce the frequencies of illegal scenarios. Some of the problem statements are discussed below.

\section{A. Sand Mining}

Sand mining is the extraction of sand, mainly through an open pit but sometimes mined from beaches and inland dunes or dredged from ocean and river beds. Sand mining is a direct cause of erosion, and impacts the local wildlife. Removal of physical coastal barriers, such as dunes, sometimes leads to flooding of beachside communities, and the destruction of beaches causes tourism to dissipate. Sand mining is regulated by law in many places, but is often done illegally. [3] Sand mining is a practice that is becoming an environmental issue in India. Environmentalists have raised public awareness of illegal sand mining in the states of Maharashtra, Madhya Pradesh, Goa and Kerala of India. [4] Conservation and environmental NGO Awaaz Foundation filed a public interest litigation in the Bombay High Court seeking a ban on mining activities along several coasts. [5] Taking a recent and local scenario as an instance, sand mining is a serious issue of environmental concern in the Kerala province of India. Even though sand mining is banned in most parts of Kerala, it is going on secretly because of the big demand of sand in the booming construction of the state.

\section{B. Child And Women Abuse}

It was found that there is an alarming rate of increase in the sexual abuse of girl children in Kerala. Child abuse takes several forms such as physical abuse, emotional/psychological abuse, sexual abuse and neglect. During the last 10 years, the public concern about child abuse and neglect has grown dramatically. From the reported cases it has to be noted that sexual abuse manifestations occurred at two levels mainlyhome and institutional level. [7] Nowadays media play an important role in bringing about such issues to the public but at the same time it has a negative impact when they merely sensationalize it. It is high time to open our eyes to initiate legal and therapeutic interventions for the protection of our children and thereby making the future of our nation bright. Domestic violence against women continues to rise in the state in spite of the introduction of the Protection of Women from Domestic Violence Act in 2005. Majority of the victims are daily wage labourers followed by housewives. Many cases are being settled out of court and most of the women approach the protection officer after registering complaints with the police.[6] Money and muscle power drowns down the justice and the offender is set free. But with solid evidences and immediate actions the culprit can be brought into justice. Recent cases of public harassments faced by women also points out to a need of a more quick and modern solution.

\section{CONCLUSION}

With our proposed system here, one can easily report the above mentioned and similar illegal acts by capturing these activities and sharing its location to the concerned authority.This will seize the crime red-handed and will indeed serve as a concrete evidence in the court of justice. We believe that this system could be a great asset to both citizens and city officials for quickly spotting a crime and preventing, or at least alleviating it.

\section{REFERENCES}

[1] Youngtae Jo and Seungki Ryu ,Highway research institute, Korea Institute of Civil Engineering and Building Technology Sensors 2015, 15, 29316-29331; doi:10.3390/s151129316

[2] DIMPLE S,MONICA V,ANIRUDH ASHOK, ADARSH C1,2,3,4Department of Information Science, PESIT Bangalore South Campus, Bangalore, India

[3] Jakob Eriksson, Lewis Girod, Bret Hull,Ryan Newton, Samuel Madden, Hari BalakrishnanMIT Computer Science and Artificial Intelligence Laboratory

[4] Aniket Kulkarni1, Nitish Mhalgi2, Sagar Gurnani3, Dr. Nupur Giri41,2,3,4V.E.S. Institute of Technology, Mumbai-74 ISSN 2250- 2459,ISO 9001:2008 Certified Journal,Volume 4, Issue 7, July 2014

[5] Manisha Mandal, Madhura Katageri, Mansi Gandhi, Navin Kore- gaonkarand Prof. Sharmila Sengupta Department of Computer Engineer- ing, Vivekanand Education Society's Institute of Technology, Mumbai

[6] 5. B, Viju (25 Sep 2010) bans sand mining across Maharashtra. Times of India. Retrieved 22 April 2013.

[7] https://en.wikipedia.org/wiki/Sand_mining in Kerala 